hep-th/9609071

HUTP-96/A042

RU-96-79

OSU-M-96-22

\title{
Small Instantons, del Pezzo Surfaces and Type I' theory
}

\author{
Michael R. Douglas ${ }^{1}$, Sheldon Katz ${ }^{2}$ and Cumrun Vafa ${ }^{1,3}$ \\ ${ }^{1}$ Department of Physics and Astronomy \\ Rutgers University \\ Piscataway, NJ 08855-0849 \\ 2 Department of Mathematics \\ Oklahoma State University \\ Stillwater, OK 74078, USA \\ 3 Lyman Laboratory of Physics \\ Harvard University \\ Cambridge, MA 02138, USA \\ mrd@physics.rutgers.edu \\ katz@math.okstate.edu \\ vafa@string.harvard.edu
}

Samll instantons of exceptional groups arise geometrically by a collapsing del Pezzo surface in a CY. We use this to explain the physics of a 4-brane probe in Type $\mathrm{I}^{\prime}$ compactification to 9 dimensions.

September 1996 


\section{Small Instantons, D-branes and del Pezzo surfaces}

It was once thought that the low energy limit of a string theory was necessarily a field theory. This may turn out to be true, but a number of examples have emerged from recent developments in string theory, for which it is unclear whether a conventional field theory description exists. Whether it does or not, string theory sheds new light on these phenomena.

An interesting example is the world-volume theory of a small $E_{8}$ instanton. In ten dimensions, this is a six-dimensional theory, apparently involving a self-dual tensor multiplet and 'tensionless strings.' In lower dimensions, $E_{8}$ symmetry can be broken, and generalizations to $E_{n}$ instantons (for $n<8$ ) appear. These theories have been studied from various viewpoints [1, 2, 3, 4, 4, 5, 6, [7].

Recently Seiberg has explained a number of features of the $E_{n}$ gauge theories in nine dimensional compactification of type $\mathrm{I}^{\prime}$ theory, as properties of the five-dimensional gauge theory of the D4-brane in type $\mathrm{I}^{\prime}$ theory [8]. The main aim of this note is to explain the observations there in the framework of 4 .

We understand that related results have been obtained and will appear in [9].

\subsection{Six dimensions}

We start with the M-theory description of small $E_{8}$ instantons [1]. Consider the Mtheory realization of $E_{8} \times E_{8}$ heterotic strings as compactifications of M-theory on an interval (identified with $S^{1} / Z_{2}$ ) [10]. Each $E_{8}$ lives on a 9-brane at the boundary of the interval. M-theory has a 5-brane, whose world-volume carries a self-dual tensor multiplet of chiral $N=2$ six-dimensional supersymmetry. When we bring it near the 9-brane, additional degrees of freedom come down, which respect $N=1$ supersymmetry. In the limit that it sits at the boundary, it can be identified with an $E_{8}$ instanton of zero size. A new branch of its moduli space appears, on which it remains on the boundary and fattens up to an $E_{8}$ instanton of finite size. By analogy with ordinary gauge theory, the branch where the five-brane is away from the boundary is a Coulomb branch, with unbroken tensor gauge symmetry. The honest instanton is the five-brane on its Higgs branch, in which the tensor is no longer massless. Instead, there are 29 new hypermultiplets describing the moduli of an $E_{8}$ instanton.

The same process has an realization in F-theory compactified on a CY 3-fold [2] [3] [4]. We consider a point on the CY moduli space where a special 4-cycle shrinks to a point. This 4-cycle is a 2 complex dimensional manifold $\mathbf{B}_{n}$ known as an $E_{n}$ del Pezzo surface 1

1 As we will note later, the root lattice of $E_{n}$ arises geometrically if $n \geq 3$. In addition, $\mathbf{P}^{1} \times \mathbf{P}^{1}$ is also a del Pezzo surface. 
- we will describe this in more detail below, but it is just $\mathbf{P}^{2}$ blown up at $n \leq 8$ points. It was conjectured in [4] that these correspond to small $E_{n}$ instantons.

The transition is described in detail in [4] where it was shown that in the context of F-theory the natural starting point is $\mathbf{B}_{9}, \mathbf{P}^{2}$ blown up at 9 points (the 9 -th point being determined by the condition that all points lie on the intersection of two cubics in $\mathbf{P}^{2}$ ). If we have a $\mathbf{B}_{k}$ shrinking to a point then we have to get rid of $9-k 2$-cycles first. This can be done by flops, where one shrinks $\mathbf{P}^{1}$ in the del Pezzo to zero size and one grows another $\mathbf{P}^{1}$ in the CY 3-fold which does not lie on the del Pezzo, going from $\mathbf{B}_{k}$ to $\mathbf{B}_{k-1}$.

In the context of F-theory, one is looking at elliptically fibered CY 3-folds with zero size fiber [11]. In this limit, all the $\mathbf{B}_{k}$ transitions get mapped to the $\mathbf{B}_{8}$ transition, and moreover the one flop needed to go from $\mathbf{B}_{9}$ to $\mathbf{B}_{8}$ gets identified with the transition point itself.

\subsection{Five dimensions}

Now we compactify an additional circle. M-theory reduces to type IIa string theory, now compactified on the $S^{1} / Z_{2}$ orientifold, with 16 D8-branes [12,13]. This is usually referred to as type $\mathrm{I}^{\prime}$ theory as it is T-dual to type I string theory on $S^{1}$. The $S O(32)$ Wilson lines on this circle translate directly into the positions of the D8-branes, and the vacuum with unbroken $S O(32)$ has all 16 D8-branes at a single boundary.

The configuration with $E_{8} \times E_{8}$ gauge symmetry has 7 D8-branes at each boundary, and another D8-brane at a distance $\phi_{0}$ from each boundary. The total length of the interval we identify with type $\mathrm{I}^{\prime}$ radius $R$. The inverse coupling constant at distance $\phi>0$ from the boundary behaves as $1 / g^{2}=\phi$ for $\phi \leq \phi_{0}$. For $\phi>\phi_{0}$, the coupling stops running and is given by $1 / g^{2}=\phi_{0}$ until it reaches the D8-brane at $R-\phi_{0}$.

To make contact with M-theory, note that in the strong coupling limit $g \rightarrow \infty$ we have $\phi_{0} \rightarrow 0$, and the one D8-brane meets the 7 D8-branes on the boundary. We can view the finite coupling configuration as a 'quantum splitting' of the 9-brane of M-theory.

The compactification reduces the 5-brane to the D4-brane of the type I' string. This is the T-dual of the D5-brane of type I string theory, and as such has an $S p(1)=S U(2)$ world-volume gauge theory. Its distance from the boundary $\phi$ is the T-dual of an $S p(1)$ Wilson line, and thus $\phi \neq 0$ breaks the $S p(1)$ to $U(1)$. Thus the Coulomb branch of the small instanton theory is the conventional Coulomb branch of the D4-brane [1].

Now, as the D4-brane moves towards the boundary, it will first hit the D8-brane which splits from the boundary. At this point, an open string stretched between the 4-brane and the D8-brane becomes massless. This is the first new physics visible in the compactification to five dimensions. 
Is this in accord with the F-theory description? This case has been analyzed in some detail in [4, 6]. The compactification of F-theory on a circle of radius $R$, using the duality chain in [11], is equivalent to M-theory on the same elliptically fibered CY 3-fold, but with an elliptic fiber of size $1 / R$. Now, the $E_{8}$ del Pezzo transition as described above is a two step procedure, where we first undergo a flop from $\mathbf{B}_{9}$ to $\mathbf{B}_{8}$, and then shrink $\mathbf{B}_{8}$ to zero size. At the first flop, the mass of the hypermultiplet obtained by wrapping the M-theory membrane around the $S^{2}$ vanishes [3].

The two descriptions are in perfect accord! In fact, this is a particular case of the equivalence (using T/S dualities) of the two descriptions of massless hypermultiplets, wrapped D-branes and open string stretched between D-branes, found in [14, 15].

This can also be extended to transitions involving higher del Pezzos. If we split off extra D8-branes from the boundary, then as the position of the D4-brane passes through each one, it corresponds to a flop in the Calabi-Yau. Let $0 \leq \phi_{i}$ for $i=1, \ldots, k-1$ denote the position of $k-1$ D8-branes, ordered so that $\phi_{i} \leq \phi_{j}$ for $i<j$.

Then the gauge coupling of the 4-brane can be derived either from the dilaton background produced by the 8-branes [12], or from the quantum dynamics of the 4-brane field theory [8]. The latter description adds to the bare coupling the one loop gauge coupling renormalization produced by the $S U(2)$ gauge multiplet and by $S U(2)$ doublet hypermultiplet matter from strings stretched between D4-brane and the D8-branes. These have masses $\left|\phi \pm \phi_{i}\right|$, and in five-dimensional gauge theory each will produce a linear correction to the gauge coupling. The result is

$$
\begin{aligned}
\frac{1}{g^{2}} & =\frac{1}{g_{0}^{2}}+8 \phi \quad \phi<\phi_{1} \\
& =\frac{1}{g_{0}^{2}}+8 \phi_{1}+7\left(\phi-\phi_{1}\right) \quad \phi_{1}<\phi<\phi_{2} \\
& \ldots \\
& =\frac{1}{g_{0}^{2}}+8 \phi_{1}+\sum_{n=2}^{k-1}(9-n)\left(\phi_{n}-\phi_{n-1}\right)+(9-k)\left(\phi-\phi_{k-1}\right) \quad \phi_{k-1}<\phi
\end{aligned}
$$

where $\frac{1}{g_{0}^{2}}$ is the coupling at the boundary. The $\phi_{i}$ are identified with the masses for the $k-1$ doublets of $S U(2)$. The limit of enhanced $E_{k}$ symmetry is $\left.\frac{1}{g^{2}}\right|_{\phi=0}=0$ and $\phi_{i}=0$ for $i=0, \ldots, k-1$. In other words the zero size $E_{k}$ instanton would correspond to $S U(2)$ at infinite coupling coupled to $k-1$ massless doublets.

We would like to see this detailed description of the gauge theory in the del Pezzo set up. In order to do this we will discuss some mathematical facts about the del Pezzos in the next section. The main result is that $\mathbf{P}^{2}$ blown up at $k$ points can be represented as a 
fibration over $\mathbf{P}^{1}$ with the fibers being generically $\mathbf{P}^{1}$ and where at $k-1$ points over $\mathbf{P}^{1}$ we have two $\mathbf{P}^{1}$ 's intersecting at one point; in other words over $k-1$ points we will have the geometry of a resolved $A_{2}$ singularity. We will show these facts in the next section, and then use them to recover the gauge theory description given above.

\section{2. del Pezzos as fibered spaces over $\mathbf{P}^{1}$}

Recall that $\mathbf{B}_{k}$ del Pezzo corresponds to $\mathbf{P}^{2}$ blown up at $k$ points. For the case of $k=1$ there are two del Pezzos, one corresponding to $\mathbf{P}^{2}$ blown up at 1 point which we denote by $\mathbf{B}_{1}$ and the other corresponding to $\mathbf{P}^{1} \times \mathbf{P}^{1}$. We show that for $k \geq 1$ this space can be given a description in terms of a fiber space over $\mathbf{P}^{1}$.

The construction we will give can be described iteratively. We will start with $\mathbf{B}_{1}$ which is obtained by blowing up a point $p$ of $\mathbf{P}^{2}$. This is the Hirzebruch surface $\mathbf{F}_{1}$ which is well known to admit a $\mathbf{P}^{1}$ fibration description. This fibration can be described concretely as follows: We pick a line $\ell \subset \mathbf{P}^{2}$ (recall the algebraic geometry terminology: a line is the same as a $\mathbf{P}^{1}$ ) not containing $p$. The fibration $\pi: F_{1} \rightarrow \ell \simeq \mathbf{P}^{1}$ is defined by sending $q \in F_{1}$ to the point of intersection of the line $p q$ with $\ell$. The fibers of $\pi$ are identified with the lines of $\mathbf{P}^{2}$ passing through the $p$. Note that this is well defined, even when $q$ lies on the exceptional divisor of the blown up point $p$. This is precisely because we have blown up the point $p$ which basically means we have replaced it by a sphere worth of points. This can also be thought of as the point $p$ together with a tangent vector at $p$. The tangent vector can be used to define the line corresponding to the fiber which again intersects the base $\ell$ at some point. Thus we have described $\mathbf{B}^{1}$ as a space whose base is $\mathbf{P}^{1}$ and whose fiber is also $\mathbf{P}^{1}$.

We can easily generalize this to $\mathbf{B}_{k}$. Now pick $k$ points $p_{1}, \ldots, p_{k}$ and blow up all these points. We apply the above construction with $p=p_{1}$. The only new thing to consider is what happens when $q$ lies on the points $p_{i}$. Clearly this gets mapped to the point on $\ell$ where the line $p_{1} p_{i}$ intersects it. Let us call it $q_{i}$. However if the point $q$ lies on the exceptional divisor of the blowup $p_{i}$ that will also map to the intersection point of $p_{1} p_{i}$ with $\ell$ which is $q_{i}$. Thus the fiber above $q_{i}$ consists of two $\mathbf{P}^{1}$ 's, one corresponding to the line $p_{1} p_{i}$ and the other coresponding to the exceptional divisor of the blowup at $p_{i}$. Note that these two $\mathbf{P}^{1}$ 's intersect at one point. Generically $q_{i}$ are distinct points on the base. Thus what we have shown is that $\mathbf{B}_{k}$ can be viewed as a fiber space over $\mathbf{P}^{1}$ where the generic fiber is $\mathbf{P}^{1}$, but over $k-1$ points the fiber has two $\mathbf{P}^{1}$ 's intersecting at a point, i.e. the geometry of a resolved $A_{2}$ singularity.

It is important for the application we have in mind to describe the Kähler classes explicitly. The dimension of $H^{1,1}\left(\mathbf{B}_{k}\right)$ is $k+1$. It is convenient to characterize the dual 
cycles as follows. Let us denote the class of the base of the above fibration by $[B]$ and the class for the generic fiber by $\left[A_{1}\right]$. Over the $q_{i}$ exceptional points on the base we have $A_{2}$ resolved singularity which has two classes associated with it for each of the blow up modes for the $\mathbf{P}^{1}$ 's. Let us denote these two classes by $\left[A_{2}^{i, 1}\right]$ and $\left[A_{2}^{i, 2}\right]$. These classes are not independent of $\left[A_{1}\right]$. In fact for each $i$ we have

$$
\left[A_{1}\right]=\left[A_{2}^{i, 1}\right]+\left[A_{2}^{i, 2}\right]
$$

To see this note that in the neighborhood of each $q_{i}$ we have a breaking of $A_{2}$ to $A_{1}$ [16]: $S U(3) \rightarrow S U(2) \times U(1)$. If we identify the two simple roots of $S U(3)$ by $e_{1}, e_{2}$, then the simple root of $S U(2)$ can be identified with $e_{1}+e_{2}$ which gives the relation above. Thus from two $\mathbf{P}^{1}$ 's over each $q_{i}$ we get only one new class which we will define as

$$
\left[A_{2}^{i}\right]=\left[A_{2}^{i, 1}\right]-\left[A_{2}^{i, 2}\right]
$$

We have thus given a basis for the $H_{2}\left(\mathbf{B}_{k}\right)$ consisting of one class of base $[B]$ one class of the generic fiber $\left[A_{1}\right]$ and $k-1$ classes $\left[A_{2}^{i}\right]$ of the difference of fiber $\mathbf{P}^{1}$ 's over $q_{i}$. Let $\omega$ denote the Kahler form on the Calabi-Yau pulled back to the del Pezzo. We can parametrize the Kahler class by $k+1$ real numbers which we denote by

$$
\phi=\omega\left(\left[A_{1}\right]\right), \quad \phi_{i}=\omega\left(\left[A_{2}^{i}\right]\right) \quad k_{B}=\omega([B])
$$

With no loss of generality we can take $\phi_{i}$ to be postive (if necessary by redefining the order of the difference of the classes).

Note that $\phi_{i}<\phi$. This is because $\omega\left(\left[A_{2}^{i, 1}\right]\right)$ and $\omega\left(\left[A_{2}^{i, 2}\right]\right.$ are positive numbers whose sum is $\phi$ and difference (in absolute value) is $\phi_{i}$. Moreover precisely when $\phi_{i}=\phi$ one of the $\mathbf{P}^{1}$ s $\left[A_{2}^{i, 2}\right]$ has shrunk to zero size. At this point the fiber over $q_{i}$ is a single $\mathbf{P}^{1}$. That is a point in moduli where we can do a flop in the Calabi-Yau where after shrinking this $\mathbf{P}^{1}$ another $\mathbf{P}^{1}$ in the Calabi-Yau, not contained in the del Pezzo grows. We continue to call the corresponding Kahler class in the Calabi-Yaul by $\phi_{i}$ even for $\phi_{i}>\phi$. In this process the del Pezzo $\mathbf{B}_{k}$ has been replaced by $\mathbf{B}_{k-1}$. As will be described in more detail in the next subsection, in doing a flop at each stage we have two choices which are equivalent except when we get to $\mathbf{B}_{2}$. At this point the two choices for the flop lead to the two different del Pezzos $\mathbf{P}^{1} \times \mathbf{P}^{1}$ and $\mathbf{B}_{1}$. Beyond this point only $\mathbf{B}_{1}$ can undergo a further flop to $\mathbf{P}^{2}$. We cannot do a further flop on $\mathbf{P}^{1} \times \mathbf{P}^{1}$.

2 Here we are assuming all the classes in the del Pezzo will be realized non-trivially in the Calabi-Yau. Otherwise there will be some restrictions on the values of the $\phi_{i}, \phi, k_{B}$. 


\subsection{More Mathematical Facts about del Pezzos}

It would be interesting to see how unique is our choice of describing del Pezzos as spaces fibered over $\mathbf{P}^{1}$. We will be examining fibrations $\pi: \mathbf{B}_{n} \rightarrow \mathbf{P}^{1}$ whose general fiber is $\mathbf{P}^{1}$. Such fibrations are characterized completely by the cohomology class of the fiber $f$. The cohomology of $\mathbf{B}_{n}$ is well known, and has as a basis the class $\ell$ of a line in $\mathbf{P}^{2}$, and the exceptional divisors $E_{i}$ of the blown up points. The canonical class of $\mathbf{B}_{n}$ is $K_{\mathbf{B}_{n}}=-3 \ell+\sum E_{i}$, and the first chern class of $\mathbf{B}_{n}$ is given by $c_{1}=-K_{\mathbf{B}_{n}}=3 \ell-\sum E_{i}$. The characterizing property of del Pezzo surfaces says that $c_{1}$ is ample, so has positive intersection with every effective curve on $\mathbf{B}_{n}$.

If $f$ is the cohomology class of the fiber of $\pi$, then we have $f^{2}=0$, and $f \cdot c_{1}=2$ by the adjunction formula. An example of such a class is $f=\ell-E_{1}$. The fibers of the corresponding projection map are lines through the origin as described above.

We now review the Weyl group $W$ of $\mathbf{B}_{n}$. A root is a cohomology class $v$ with $v^{2}=-2$ and $v \cdot c_{1}=0$. To each root corresponds a reflection $\rho_{v}$ on the cohomology

$$
\rho_{v}(D)=D+(D \cdot v) v
$$

Note that $\left(\rho_{v}(D)\right) \cdot\left(\rho_{v}\left(D^{\prime}\right)\right)=D \cdot D^{\prime}$ and $\left(\rho_{v}(D)\right) \cdot c_{1}=0$. This implies that each $\rho_{v}$ preserves the orthogonal complement $V$ of $c_{1}$, which together with the set of all roots form a root system. We let $W=W\left(\mathbf{B}_{n}\right)$ be the Weyl group of this root system. Note that $W$ acts as a group of isometries of the cohomology of $\mathbf{B}_{n}$.

We have the following table.

$\begin{array}{ccc}n & \text { roots } & \text { root system } \\ 1 & \text { none } & \text { none } \\ 2 & E_{i}-E_{j} & A_{1} \\ 3 \leq n \leq 8 & \text { generated by }\left\{E_{i}-E_{j}, \ell-E_{i}-E_{j}-E_{k}\right\} & E_{n}\end{array}$

In the above, the root systems $E_{3}, E_{4}, E_{5}$ respectively denote $A_{2} \times A_{1}, A_{4}, D_{5}$.

Recall that a -1 curve is a curve $C$ with $C^{2}=-1$ and $C \cdot c_{1}=1$. These curves are well known. They are of one of the following types.

$$
\begin{array}{cc}
E_{i} & n \geq 2 \\
\ell-E_{i}-E_{j} & n \geq 5 \\
2 \ell-\sum_{k=1}^{5} E_{i_{k}} & n \geq 7 \\
3 \ell-2 E_{i}-\sum_{k=1}^{6} E_{i_{k}} & n=8 \\
4 \ell-2 \sum_{k=1}^{3} E_{i_{k}}-\sum_{m=1}^{5} E_{j_{m}} & n=8 \\
5 \ell-2 \sum_{k=1}^{6} E_{i_{k}}-\sum_{m=1}^{2} E_{j_{m}} & n=8 \\
6 \ell-3 E_{1}-2 \sum_{k=1}^{7} E_{i_{k}} & n=8
\end{array}
$$


We now show that any fibration with general fiber $\mathbf{P}^{1}$ can be brought to this form by an application of an element of $W$. That is, we will show that there exists a $w \in W$ such that $w \cdot f=\ell-E_{1}$.

Since the Euler characteristic of $\mathbf{B}_{n}$ is $n+3$, and $\mathbf{P}^{1}$ fibrations over $\mathbf{P}^{1}$ have Euler characteristic 4 , we see that not all fibers can be $\mathbf{P}^{1}$ (except in the case $n=1$ ). Since $c_{1}$ is an ample class, we see that if a reducible fiber $f$ splits up into two pieces $f=f_{1}+f_{2}$, we necessarily have $f_{i} \cdot c_{1}=1$ for $i=1,2$. Since $f_{i}$ must be rational, the adjunction formula gives $f_{i}^{2}=-1$. Also, a fiber cannot be a multiple fiber, since it would necessarily have multiplicity $2, f=2 g$ with $g \cdot c_{1}=0$; but this contradicts the adjunction formula.

If $f=f_{1}+f_{2}$ as above, we have $f_{i} \cdot f=0$, from which we compute $f_{1} \cdot f_{2}=1$. So the only fibers which are not $\mathbf{P}^{1} \mathrm{~s}$ are a pair of intersecting $\mathbf{P}^{1} \mathrm{~s}$ forming an $A_{2}$ configuration. Calculation of the Euler characteristic of $\mathbf{B}_{n}$ from this geometry shows that there must be $n-1$ singular fibers of type $A_{2}$.

We now classify these fibrations. If $n=1$, then it is easy to see from $f^{2}=0$ and $f \cdot c_{1}=2$ that the only such fibration has $f=\ell-E_{1}$. If $n=2$, we similarly see that the only fibrations have $f=\ell-E_{1}$ and $f=\ell-E_{2}$, and these are permuted by the reflection in the root $v=E_{1}-E_{2}$. If $n>2$, then there is a singular fiber consisting of two intersecting -1 curves. It is well known that we can use the Weyl group to bring $f_{1}$ to $E_{2}$. We will show that we can then choose an element of the Weyl group which fixes $E_{2}$ and brings $f_{2}$ to $\ell-E_{1}-E_{2}$; this brings $f$ to $\ell-E_{1}$ as claimed.

From the classification of -1 curves described above, the only ones that meet $E_{2}$ once are

$$
\begin{gathered}
\ell-E_{2}-E_{i}, \\
2 \ell-E_{2}-\sum_{k=1}^{4} E_{i_{k}}, \quad(n \geq 5), \\
3 \ell-E_{2}-\sum_{k=1}^{5} E_{i_{k}}-2 E_{i}, \quad(n \geq 7), \\
4 \ell-E_{2}-\sum_{k=1}^{4} E_{i_{k}}-2 \sum_{j=1}^{3} E_{m_{j}}, \quad(n=8), \\
5 \ell-E_{2}-E_{i}-2 \sum_{k=1}^{6} E_{i_{k}}, \quad(n=8),
\end{gathered}
$$

where in each line, any fixed exceptional divisor appears at most once. 
It is straightforward in each of the cases above to find a series of reflections which achieves the desired result. For a class of the first type listed above, we can reflect in the root $E_{1}-E_{i}$. In fact, each of the classes in the above list can be brought to the form of a class listed immediately above it by a simple reflection.

We thus conclude that the description of the del Pezzos as fiber spaces over $\mathbf{P}^{1}$ is unique up to the action of the Weyl group.

As an example of the use of the Weyl group, suppose we flop the exceptional curve $\ell-E_{1}-E_{2}$. This replaces $\mathbf{B}_{n}$ by $\mathbf{B}_{n-1}, \frac{3}{3}$ and preserves the fibration with fiber $\ell-E_{1}$. However, due to the blowdown, $E_{1}$ is no longer an exceptional curve; we have $E_{1}^{2}=0$ in the blown down surface. To make contact with our standard description of the fibration, we choose a root, say $v=\ell-E_{1}-E_{2}-E_{n}$, which has been chosen so that $\rho_{v}\left(E_{n}\right)=$ $\ell-E_{1}-E_{2}$. The exceptional curves $E_{i}^{\prime}=\rho_{v}\left(E_{i}\right), 1 \leq i \leq n-1$ are $n-1$ disjoint exceptional curves which are disjoint from $\ell-E_{1}-E_{2}$, hence remain exceptional after the flop. These $n-1$ curves may be identified with exceptional divisors of $\mathbf{B}_{n-1}$. Putting $\ell^{\prime}=\rho_{v}(\ell)=2 \ell-E_{1}-E_{2}-E_{n}$, we see that the fibration has fiber $\ell^{\prime}-E_{1}^{\prime}=\ell-E_{1}$, and we may continue our analysis through these flop transitions if desired.

\subsection{Generalized del Pezzos}

It is known that it is possible for singular surfaces $S$ to be contained in smooth CalabiYau threefolds and be contractible to a point. Conditions for these "generalized del Pezzo surfaces" are given in [17]. We list a few of these conditions.

1. $S$ is Gorenstein (roughly speaking, the singularities of $S$ are mild enough so that the canonical bundle of the smooth part of $S$ extends across the singularities to a bundle $\omega_{S}$ on all of $S)$.

2. $\omega_{S}^{*}$ is ample.

3. The reduced, irreducible components of $S$ are surfaces of degree $a-1$ or $a$ in $\mathbf{P}^{a}$, and in particular are either rational or elliptic ruled surfaces.

If $S$ is smooth, then the only possibilities are $S=B_{n}(0 \leq n \leq 8)$ or $S=\mathbf{P}^{1} \times \mathbf{P}^{1}$. Here we give three examples of generalized del Pezzos which are not smooth and illustrate the above conditions.

Example 1. Let $C$ be a plane cubic curve, for example $x^{3}+y^{3}+z^{3}=0$. We now view the same equation as an equation in $\mathbf{P}^{3}$ with an extra coordinate $w$. The resulting surface $S$

3 This is only true if $n \geq 3$; if $n=2$, then we get $\mathbf{P}^{1} \times \mathbf{P}^{1}$. 
has a singularity at $(1,0,0,0)$, and $\omega_{S}^{*}$ is just the restriction of the ample class $\mathcal{O}(1)$ from $\mathbf{P}^{3}$ to $S$. This is an example of an elliptic ruled surface.

This $S$ can be put inside a smooth Calabi-Yau. For example, the equation

$$
x^{3}+y^{3}+z^{3}+f_{4}(w, x, y, z)+f_{5}(w, x, y, z)=0
$$

is the affine form of the equation of a quintic in $\mathbf{P}^{4}$, where $f_{i}$ denotes a general polynomial of degree $i$. It is singular at $(1,0,0,0)$. We blow up the singularity by replacing $(x, y, z)$ by $(x w, y w, z w)$ and obtain

$$
x^{3}+y^{3}+z^{3}+w f_{4}(1, x, y, z)+w^{2} f_{5}(1, x, y, z)=0 .
$$

This is now smooth due to the presence of a linear term from $w f_{4}$. The exceptional divisor is defined by $w=0$, and is identified with the surface $S$ just described.

Example 2. Here we take $S \subset \mathbf{P}^{3}$ to be a general cubic hypersurface with an $A_{1}$ singularity. Again, $\omega_{S}^{*}$ is just the restriction of the ample class $\mathcal{O}(1)$ from $\mathbf{P}^{3}$ to $S$. This $S$ can be put inside the blowup of a quintic in a similar way, replacing $x^{3}+y^{3}+z^{3}$ above by $w\left(x y+z^{2}\right)+f_{3}(w, x, y, z)$, with $f_{3}$ a general cubic.

This example is interesting in that it admits a $\mathbf{P}^{1}$ fibration. One way to see this is that it is a degenerate version $\tilde{B}_{6}$ of $B_{6}$. The 6 points of $\mathbf{P}^{2}$ to be blown up are chosen to lie on a conic $C$, which therefore is in the class $2 \ell-\sum E_{i}$ on $\tilde{B}_{6}$. Then $c_{1}$ is no longer ample since $c_{1} \cdot C=0$. Since $C^{2}=-2$, shrinking $C$ yields an $A_{1}$ singularity on a surface $S$. It can be shown that any cubic hypersurface with an $A_{1}$ singularity is of this form, with $c_{1}$ corresponding to the hyperplane class [18].

We need to find a fibration $\pi: \tilde{B}_{6} \rightarrow \mathbf{P}^{1}$ such that the conic $C$ is contained in the fiber; this guarantees that after collapsing $C$ to a point, we still have a fibration $S \rightarrow \mathbf{P}^{1}$. This can be achieved by taking $f=2 \ell-E_{1}-E_{2}-E_{3}-E_{4}$ (which arises from reflection of our standard fiber by the root $v=\ell-E_{2}-E_{3}-E_{4}$ ). From the Weyl group action, we might have expected $5=6-1$ fibers of type $A_{2}$ as before. But something interesting happens. We expect $A_{2}$ fibers to arise by applying the Weyl group to $\left(E_{5}\right)+\left(\ell-E_{1}-E_{5}\right)$ and $\left(E_{6}\right)+\left(\ell-E_{1}-E_{6}\right)$. But we have

$$
\begin{array}{ccccc}
\rho_{v}\left(E_{5}\right) & = & E_{5} & & \\
\rho_{v}\left(E_{6}\right) & = & E_{6} & \\
\rho_{v}\left(\ell-E_{1}-E_{5}\right) & = & 2 \ell-E_{1}-E_{2}-E_{3}-E_{4}-E_{5} & = & C+E_{6} \\
\rho_{v}\left(\ell-E_{1}-E_{6}\right) & = & 2 \ell-E_{1}-E_{2}-E_{3}-E_{4}-E_{6} & = & C+E_{5}
\end{array}
$$


This says that the expected $A_{2}$ fibers $\rho_{v}\left(E_{5}\right)+\rho_{v}\left(C+E_{6}\right)$ and $\rho_{v}\left(E_{6}\right)+\rho_{v}\left(\ell-E_{1}-E_{6}\right)$ coincide on $\tilde{B}_{6}$; they are each $C+E_{5}+E_{6}$, an $A_{3}$. H The curve $C$ lies in the middle of the $A_{3}$, so after contracting, we get an $A_{2}$ conisting of $E_{5}+E_{6}$ passing through the singular point of $S$. We reach a situation with 4 distinct $A_{2}$ fibers, one of which having its intersection point precisely passing through the singular point of $S$.

Example 3. For an example with more than one component, consider $S=\mathbf{P}^{1} \times A_{2}$. Here, $S$ has two components $S_{1}$ and $S_{2}$, each isomorphic to $\mathbf{P}^{1} \times \mathbf{P}^{1}$. Each component has second cohomology $\mathbf{Z}^{2}$, and we write cohomology classes as integers $(a, b)$. A class is ample if and only if $a>0$ and $b>0$. We represent the class of the curve $S_{1} \cap S_{2}$ as $(0,1)$ (in either $S_{1}$ or $\left.S_{2}\right)$. The $\omega_{S}^{*}$ restricts to each $S_{i}$ as the class $(2,1)$, hence is ample.

By degenerating $\mathbf{P}^{1}$ to $A_{2}$, we get a degeneration of $\mathbf{P}^{1} \times \mathbf{P}^{1}$ to $S$. No flops would be involved in such a transition.

\section{3. del Pezzo and $S U(2)$ gauge theory}

If we consider type IIA theory or M-theory on $K 3$ near an $A D E$ singularity, it is well known that we end up with $A D E$ gauge symmetry in 6 or 7 dimension respectively. Moreover it was argued by employing the adiabatic argument [19, 20,21,22] that even if we consider Calabi-Yau 3-fold compactifications down to 4 or 5 dimensions, whenever we have a singularity over a surface of $A D E$ type we obtain $A D E$ gauge symmetry with $N=2$ supersymmetry in 4 dimensions or $N=1$ in 5 dimensions. Moreover if the genus of the corresponding surface is $g$ we obtain $g$ adjoint hypermultiplet matter in addition [21] [22]. If over the base there are additional singularities we will get extra matter. Aspects of these were discussed in [23] [4] [24] [25] [16]. In particular the analysis of [16] shows that if we have an $A_{1}$ singularity fiber which at some points gets enhanced to $A_{2}$, what survives of the $S U(3)$ in this fibration is $S U(2) \times U(1)$ and the corresponding adjoint is a hypermultiplet in the doublet of $S U(2)$ and charged under the $U(1)$.

Let us now go back to the del Pezzo $\mathbf{B}_{k}$ in view of this description. Consider the limit in which the del Pezzo has a large base $[B]$ with small fiber. In this limit using the adiabatic arguments mentioned above we immediately deduce that we have an $S U(2) \times U(1)^{k-1}$ gauge symmetry with $k-1$ doublets of $S U(2)$ charged under distinct $U(1)$ 's. Moreover going to the Coulomb branch of the $S U(2) \times U(1)^{k-1}$ theory corresponds to resolving the

4 Note that $\tilde{B_{n}}$ is an auxiliary space in the analysis, and is not contained in a Calabi-Yau. If we had an $A_{n}=C_{1}+\ldots C_{n}$ fiber in a generalized del Pezzo inside a Calabi-Yau, the adjunction formula gives $\left(C_{1}+\ldots+C_{n}\right) \cdot c_{1}\left(\omega_{S}^{*}\right)=2$; but this is only possible for an ample class if $n \leq 2$. 
generic $A_{1}$ singularity of the fiber and resolving the $k-1 A_{2}$ singular fibers. We thus immediately identify the $\phi$ defined in the previous section (the size of the $\left[A_{1}\right]$ fiber) with the Coulomb branch of $S U(2)$ and the $\phi_{i}$ with the difference in Kahler class of the two $\mathbf{P}^{1}$ 's of the fiber over $q_{i}$, which can also be identified with the mass parameters of the $k-1$ doublets (since they are charged under the $U(1)$ 's). We thus have an $S U(2)$ gauge symmetric theory in its Coulomb branch with $\phi$ as the expectation value of the scalar together with $k-1$ doublets of mass $\phi_{i}$.

The bare coupling constant of $S U(2)$ can be easily identified. Up to an overall rescaling it is proportional to the area of the base $[B]$ of the fibration. This simply follows from the fact that the effective coupling in the five dimensional theory gets a volume factor from the internal space. Thus (up to a convention dependent normalization) we identify the $S U(2)$ coupling $\frac{1}{g_{0}^{2}}$ with

$$
\frac{1}{g_{0}^{2}}=k_{B}
$$

Note that in the limit of $E_{d}$ small instanton the whole del Pezzo shrink to zero size and thus in particular $\frac{1}{g^{2}}=k_{B} \rightarrow 0$. One can also explain the running of the gauge coupling constant with respect to $\phi_{i}$ in a geometrical way. The easiest way to see this is to start from $\mathbf{B}_{9}$, where the coupling constant does not run. Then each time there is a flop the coupling runs. This comes about geometrically as follows [3]: There are interactions of the form

$$
\int C_{I J K} A^{I} F^{J} F^{k}
$$

in the Calabi-Yau compactification of M-theory [26], where $C_{I J K}$ denote the triple intersection of $H_{4}$ classes and the $A^{I}$ are the gauge fields. Each time the CY undergoes a flop the classical interesection number in that class changes and gives rise to a new interaction of the form $\int A \wedge F \wedge F$ and by supersymmetry to an interaction of the form $\int \phi F^{2}[\mathbb{Z}$. In our case, consider the kahler class $\mathcal{D}$ dual to the del Pezzo $\mathbf{B}_{k}$ sitting in the Calabi-Yau. We have

$$
D \cdot D \cdot D=9-k
$$

Note that this Kahler class is responsible for shrinking the del Pezzo and in particular corresponds to changing of $\phi$. This classical self-intersection thus induces an interaction of the form $\int(9-k) \phi F^{2}$ which is responsible for the running of the coupling constant with $\phi$. Each time there is a flop the value of $k$ changes and the dependence of the gauge coupling on $\phi$ also changes accordingly.

We have thus reproduced all the expected gauge theoretic properties of the small $E_{k}$ instantons $(k \neq 1)$ in the del Pezzo setup. As mentioned before for the case of $k=1$ case we have two choices of del Pezzo and there seems to be a puzzle of which one we get in the probe theory. We return to this point in section 5 . 


\subsection{Physics of generalized del Pezzos}

As discussed before there are cases in the compactification of M-theory on Calabi-Yau where the 4-cycle that shrink is not a smooth del Pezzo, but what is called a generalized del Pezzo. We gave a few examples of it in the previous section. It would be interesting to unravel the physics of them as one might expect that they lead to new fixed points of quantum field theories in 5 dimensions. These may lead to new physics which may not even be seen by a D4-brane probe in the simple set up we have. For instance if we consider example 3 in section 2.2, from the above considerations we expect to have an $S U(3)$ gauge theory fixed point at infinite coupling with no matter (there is a mathematical no-go theorem that suggests that $S U(3)$ with fundamental matter cannot lead to an interesting fixed point at infinite coupling). The example 2 of section 2.2 seems to correspond to $S U(2)$ with 4 fundamentals because it corresponds to an $A_{1}$ fibration over $\mathbf{P}^{1}$ where at four points we have $A_{2}$ fibration. However as discussed before one of the $A_{2}$ fibers meets the base at a singular point, suggesting something extra happens to one of the fundamentals. It would be interesting to unravel the physics of this extra singularity. The example 1 of section 2.2 does not admit a fibration description, so there is no non-abelian gauge symmetry description of this fixed point. This is similar to the $\mathbf{P}^{2}$ case.

\section{4. $D_{n}$ instantons versus $E_{n}$ instantons}

Implicit in the D-brane discussion is the fact that the small $S O(32)$ instanton and the small $E_{n}$ instanton, after compactification on $S^{1}$, are the same object in different regions of moduli space. The D4-brane which was argued to describe a small $E_{n}$ instanton is simply the T-dual of the small $S O(32)$ instanton, a D5-brane in type I theory. In particular the gauge symmetry $S U(2)$ and the matter content was deduced in this way. Any truly novel physics of small $E_{n}$ instantons will appear in the limit of infinite coupling on the D4-brane, as described in [8].

This equivalence bears some analogy to the example of tensionless strings associated with degenerating two-cycles in type IIb theory. After compactification on $S^{1}$, these are the T-duals of the massless gauge bosons of type Ila theory. One might conjecture that

such a relation between non-critical low energy string theories and more conventional field theory is general.

Since the relation between $E_{n}$ and $D_{n}$ has not been discussed explicitly, it may be worth following it in more detail. We first recall how the two heterotic strings are continuously connected [27,28]. Start with $S O(32)$; in the compactification the gauge symmetry 
is $S O(32) \times U(1)^{2}$. By turning on a Wilson line, one can break this to a subgroup such as $S O(14)^{2} \times U(1)^{4}$, and by varying the Wilson line continue to a point with $E_{8} \times E_{8} \times U(1)^{2}$.

Since there is an unbroken $S U(2)$ throughout the process, one can follow the small instanton of the $S O(32)$ theory, to produce a small instanton of the $E_{8}$ theory, which implies that the two objects are the same. Indeed there is no quantum number in the fivebrane solution of [29] available to distinguish them - the only possible distinction could be the embedding of $S U(2)$ in the gauge group, and one can check that this process produces an instanton with the same (minimal) embedding of $S U(2)$.

This leads to a bit of a paradox, as it implies that all of the strange physics of the $E_{8}$ small instanton should be implicit in the rather tame-looking $S O(32)$ small instanton. This is essentially the same paradox that appeared in the early discussion of heterotic type I duality. Duality requires that the heterotic string world-sheet physics responsible for gauge symmetry enhancement to $E_{8}$ be present in the type I string. Since it is true for any value of the heterotic string coupling including strong coupling, it must be visible at weak type I coupling, which naively rules out the possibility of solitonic states becoming light.

The resolution was that type I perturbation theory still breaks down, due to a failure of the dilaton tadpoles from the disk and $\mathbb{R} \mathbb{P}^{2}$ to cancel in winding sectors. This effect becomes important as the type I compactification radius becomes small, and indeed the region of heterotic string moduli space with enhanced gauge symmetry always maps into this regime [12]. The resulting physics is much clearer in the T-dual picture, where the dilaton becomes strong at a fixed point of the orientifold, but in principle all of these effects could be translated back to the original type I picture. For example, the D0-branes which became the gauge bosons of $E_{8}$ gauge symmetry in [13], correspond to type I heterotic solitons wrapped around a small circle.

Thus, the statement is that $D_{n-1}$ gauge symmetry in either theory can be promoted either to $D_{n}$ or to $E_{n}$ by tuning different parameters. In the type $\mathrm{I}^{\prime}$ description, $D_{n}$ is achieved by bringing another D8-brane to the boundary, while $E_{n}$ is achieved by going to infinite coupling on the boundary. A zero size instanton which is present will gain the additional massless states of $D_{n}$ or $E_{n}$ global symmetry, and the corresponding additional dimensions on its Higgs branch, thanks to the same physics.

The case of $D_{5} \cong E_{5}$ symmetry is an interesting illustration. The theory (at one boundary) has manifest $S O(8) \times U(1)^{2}$ gauge symmetry, which can be enhanced to $S O(10) \times U(1)$ in two ways. If another 8 -brane is brought to the boundary, stretched open strings in vector multiplets come down, producing $45=28_{0}+1_{0}+8_{v, 1}+8_{v,-1}$. On the other hand, by adjusting parameters to take the strong coupling limit at the boundary, 
D0-branes become massless, charged under a different $U(1)$, and with fermion zero modes (from 0-8 strings) in the vector of $S O(8)$. Quantizing these puts the 0-branes in spinor representations of $S O(8)$, to produce $45=28_{0}+1_{0}+8_{s, 1}+8_{c,-1}$.

The global symmetry on the D4-brane is also enhanced. Electrically charged matter $Q_{i}$ from the 4-8 strings transforms in the vector of $S O(8)$, and in the $D_{5}$ case becomes the vector of $S O(10)$. In the $E_{5}$ case, since the 4-brane gauge coupling is strong, it is only sensible to consider gauge singlet operators, such as the bilinears $Q_{i} Q_{j}$ in the adjoint of $S O(8)$. These must be joined by 0 -brane - 4-brane bound states to fill out the adjoint of $S O(10)$, and the simplest way this could work is if each 0-brane which appeared as a gauge multiplet can also appear as part of a unique multiplet of $0-4$ bound states. The same story could account for $E_{n}$ global symmetry, and it would be quite interesting to check it against precise results for $0-4$ bound states.

Additional 4-brane BPS states are predicted in [5],6], and it might be even more interesting to identify these, as some of them are singlet under the $E_{n}$ symmetry, and thus need not involve D0-branes.

The continuous connection between the D5-brane and the D4-brane appears to require string theory, but this is not incompatible with the idea that the non-trivial physics of the D4-brane and the origin of the $E_{n}$ massless states has a purely field theoretic explanation. The D0-branes of the string description would be equivalent to instantons of the fivedimensional gauge theory.

\section{5. $\mathbb{F}_{1}$ versus $\mathbb{P}^{1} \times \mathbb{P}^{1}$ and a discrete $\theta$ angle}

The detailed agreement between the del Pezzo description and the gauge theory description for every other case leads us to return to the puzzling example of the surfaces $\mathbb{F}_{1}$ and $\mathbb{P}^{1} \times \mathbb{P}^{1}$, and try to understand this in gauge theory terms.

Both surfaces can be realized by flopping a $\mathbb{P}^{1}$ in $B_{2}$, so let us describe the choice which distinguishes them. In general, we can flop any of the exceptional curves, and since all of them are related by the Weyl group, the result is the same. But for $B_{2}$, the curves $E_{2}$ and $\ell-E_{1}-E_{2}$ are not so related. Since $\phi$ is the size of the $A_{1}$ fiber $\ell-E_{1}$, and $\phi_{1}$ is the size of $\ell-E_{1}-2 E_{2}$, the two exceptional curves correspond to states with mass $\left(\phi-\phi_{1}\right) / 2$ and $\left(\phi+\phi_{1}\right) / 2$.

Thus, the mathematics appears to be telling us that the definition of pure $S U(2)$ supersymmetric gauge theory in five dimensions involves a subtle two-valued choice, which upon adding massless matter becomes vacuous. Starting from the theory with one matter multiplet, we can recover the pure gauge theory by taking its bare mass to infinity, but we 
will get different results depending on whether we take it to positive or negative infinity (relative to the sign of $\phi$; of course an $S U(2)$ gauge transformation flips the sign of all $\phi_{i}$ and $\phi)$.

This is very reminiscent of the way the $\theta$ angle in $d=4$ gauge theory becomes vacuous in a theory with massless fermions, and is affected by the phase of the fermion mass terms. Thus we ask whether $d=5 S U(2)$ gauge theory admits a $\mathbb{Z}_{2}$-valued $\theta$ angle.

Just as the $\theta$ angle in $d=4$ weighs gauge field configurations by $\exp i \theta n$ with $n$ the instanton number in $\pi_{3}(S U(2))$, a $\mathbb{Z}_{2} \theta$ angle in $d=5$ will exist if $\pi_{4}(S U(2)) \cong \mathbb{Z}_{2}$. Indeed this is true, and we identify this $\theta$ angle as the two-valued choice.

We next need to understand its relation to a fermion mass $m$. In $d=4$ this was a consequence of the axial anomaly. To use an argument which generalizes to the discrete case, it follows from the existence of $2 n k$ fermion zero modes in the $n$-instanton sector, requiring $n k$ insertions of the mass term $m \bar{\psi} \psi$ for a non-zero amplitude, and weighing this sector by $m^{n k}$.

Now, a known consequence of the fact that $\pi_{4}(S U(2)) \cong \mathbb{Z}_{2}$ is the global anomaly in $d=4 S U(2)$ gauge theory with an odd number of fermion doublets [30]. This is a sign ambiguity in the fermion determinant $(\operatorname{det} \not D)^{1 / 2}$ under a global gauge transformation $U$ in the non-trivial class of $\pi_{4}(S U(2))$. In [30], this ambiguity was exhibited by finding a non-trivial path in configuration space connecting a gauge field configuration $A^{(0)}$ with the configuration gauge transformed by $U$, and showing that an odd number of eigenvalues of the Dirac operator will change sign along the path. Such a path defines a five-dimensional gauge field in the non-trivial class of $\pi_{4}(S U(2))$, and this is equivalent to the statement that the mod two index for the five dimensional Dirac operator will be odd in this field configuration.

This fact also implies that in our five dimensional theory, changing the sign of a single fermion mass will flip the $\mathbb{Z}_{2}$ theta angle. Each 8-brane and its image comes with a halfhypermultiplet in the $(2,2)$ of $S O(2) \times S U(2)$, corresponding to two Weyl doublets in the $d=4$ theory (we know that the $d=4$ reduction is non-anomalous), and a fermion determinant $\operatorname{det}(\not D+m)$, defined unambiguously by combining the fermions into a single Dirac fermion. The operator $\not D$ is real antisymmetric and in a sector with $k$ fermion zero modes, the determinant is a product over eigenvalues

$$
\operatorname{det}(\not D+m)=m^{k} \prod_{i}\left(\lambda_{i}+m\right)\left(\lambda_{i}-m\right)
$$

proving the result. We thus find that the moduli space of five dimensional gauge theory mirrors the moduli space of del Pezzo surfaces in every detail. 
Now that we have shown that the distinction between $\mathbb{F}_{1}$ and $\mathbb{P}^{1} \times \mathbb{P}^{1}$ is a distinction between two different $S U(2)$ gauge theories, let us discuss the difference between the physics of the two cases. The main difference is that whereas $\mathbb{P}^{1} \times \mathbb{P}^{1}$ can only be shrunk to zero volume in a single way, $\mathbb{F}_{1}$ has two independent parameters. In particular, it admits a final flop, down to $\mathbb{P}^{2}$. Geometrically the membrane wrapped around the exceptional divisor becomes massless at this point, which implies that for $\frac{1}{g^{2}}=c \phi$ we have a massless hypermultiplet BPS state with charge 1 under the $U(1)$. Beyond this point the running of the coupling should change, as discussed before, to $1 / g^{2} \sim$ const. $-9 \phi$. Moreover beyond this point we do not have any fiber space description which implies that even at $\phi=0$ we do not have the massless $W^{ \pm}$bosons. This suggests that after the last flop the $W^{ \pm}$ become unstable.

The appearance of a new massless charged BPS state in the pure $S U(2)$ gauge theory at finite coupling is somewhat surprising and deserves explanation. Since the BPS mass formula for this state involves $1 / g^{2}$, this must be a bound state involving a D0-brane (or instanton). Indeed the total charge is that of a bound state with the massive $S U(2)$ gauge boson, and the positive instanton mass $1 / g^{2}$ is being canceled by an opposite sign central charge for the $\mathrm{W}$ boson. At this point the $W^{ \pm}$become marginally unstable to decay into the instanton BPS state and the new hypermultiplet, and the geometry as well as the running of the coupling beyond the transition indicate that they become unstable.

The point in $S^{1} / \mathbb{Z}_{2}$ where the bound state becomes massless is not associated with any feature of the background, and this might be thought to be a contradiction to the general 'probe' philosophy [31,32,33, 34] that gauge dynamics on the brane must reproduce the background fields, in this case the dilaton. Now for the discrete $\theta$ angle corresponding to $\mathbb{P}^{1} \times \mathbb{P}^{1}$, the probe philosophy works. Furthermore, we can take several probes and take some of them through the orientifold point from $\phi$ to $-\phi$, thus choosing independent $\theta$ angles for each. Thus we would associate the $\mathbb{F}_{1}$ dynamics not to a difference in the background but rather with a choice made on the 4 -brane which makes it not act as a probe. One might entertain other interpretations and perhaps more can be said about this issue.

A comment which may make the result more palatable than it seems at first is that similar results are implied by the global symmetry in the $N_{f} \geq 1$ cases. The Weyl group of the full enhanced symmetry will act on the moduli space and spectrum, and exchange points of moduli in the gauge theory parametrization which do not seem to look like symmetries of the perturbative spectrum. As discussed before the resolution is that under the Weyl group the perturbative states mix with non-perturbative solitonic objects in the field theory language, i.e. BPS instanton D0-branes. Our present discussion of the flop to $\mathbb{F}_{1}$ is just the simplest manifestation of this. 


\section{Discussion}

We found agreement between two descriptions of the Coulomb branch for $E_{n}$ zero size instantons in five dimensions, one provided by F-theory and M-theory on a Calabi-Yau containing a degenerating del Pezzo surface [4], the other provided by the five-dimensional gauge theory physics of D4-branes in type $\mathrm{I}^{\prime}$ theory [8]. We also found hints of new fivedimensional fixed point theories, for which we do not know of a D-brane description, by considering generalized del Pezzos.

We also discussed some general properties of the Higgs branch of the gauge theory, describing the true small instantons. For example, we saw that the $D_{n}$ and $E_{n+1}$ small instantons are continuously connected, and the symmetry enhancement of the infinite coupling limit is associated with additional 0-brane (or instanton) states becoming massless. Their associated fields provide the additional parameters of $E_{n+1}$ instanton moduli space, which enter on an equal footing with the $D_{n}$ parameters.

Even though many aspects of the physics associated with small $E_{n}$ instantons can be captured by gauge theory dynamics, one should keep in mind that this is at a fixed point at infinite coupling, and thus the spectrum is not evident from this description. The underlying dynamics of the theory remains somewhat mysterious. For example, the geometric description predicts a tensionless string in five dimensions, from a 5-brane wrapped around the vanishing 4-cycle [3]. This string will be as relevant as all the massless particle states coming from membranes wrapped around 2-cycles inside the del Pezzo. On the other hand, the origin of this string in the gauge theory is the 't Hooft-Polyakov monopole solution [8]. As $S U(2)$ is restored, it might be thought that this solution would delocalize and should not be considered part of the spectrum, but this is at finite coupling. It is consistent with what we know so far to imagine that at the non-trivial fixed point, it remains in the theory.

It remains to develop effective ways to discuss the physics at the fixed point. In this regard it is tempting to conjecture, given that $\frac{1}{g^{2}} \rightarrow 0$ that we are left with a pure $N=1$ Chern-Simons theory in 5 dimensions. This suggests that the relevant aspects of the fixed point may be captured by a topological supersymmetric Chern-Simons theory in 5 dimensions.

The situation will change when one compactifies one more dimension. In this case the formal scaling arguments suggest that the leading massless modes are particles [6] and there should be a more conventional field theory description. This is in fact supported by the recent study of this case [7]. We will be very brief here and only point out some basic features relevant for further study. In this case it is natural to consider the F-theory compactification on $K 3$ [11] and study a 3-brane probe following [35, 33, 36, 37]. It may appear that we will see a clash between the del Pezzo description and the 3-brane probe 
behavior near an $E_{6,7,8}$ enhanced gauge symmetry point. We expect from [36] that the corresponding gauge coupling $\tau$ on the probe is at fixed but finite values (at the fixed points of $S L(2, \mathbf{Z})$ elements of order $3,4,6)$. However the type IIA description on a CY 3-fold with a zero size del Pezzo, which is what we obtain after compactification of M-theory on circle, might seem to lead to infinite coupling as before.

We believe the resolution of this puzzle may be that for type IIA (unlike the Mtheory case), because we have corrections to the volume of Calabi-Yau due to worldsheet instantons, 5 at the transition point the quantum volume of the base (together with the $B$-field) will not be zero but instead be given by the fixed point values of $\tau$ predicted by [36]. This will be very interesting to study. In particular the results of [7] should be derivable by using mirror symmetry acting on del Pezzo (along the lines of the appendix in [6]).

We would like to thank B. Crauder, B. Greene, W. Lerche, D. Morrison, N. Seiberg and N.P. Warner for valuable discussions. C.V. would like to thank the hospitality of Rutgers University where this work was done.

The research of M.R.D. was supported in part by DOE grant DE-FG02-96ER40559, that of S.K. was supported in part by NSF grant DMS-9311386 and NSA grant MDA90496-1-0021, and that of C.V. was supported in part by NSF grant PHY-92-18167.

5 C.V. thanks B. Greene for reminding him of this in the present context. 


\section{References}

[1] O. Ganor and A. Hanany, "Small E(8) Instantons and Tensionless Non-critical Strings," hep-th/9602120.

[2] N. Seiberg and E. Witten, "Comments on String Dynamics in Six-Dimensions," hepth/9603003.

[3] E. Witten, "Transitions in $M$ Theory and $F$ Theory," hep-th/9603150.

[4] D. Morrison and C. Vafa, "Compactifications of $F$ theory on Calabi-Yau Threefolds I," hep-th/9602114; and "Compactifications of $F$ theory on Calabi-Yau Threefolds II," hep-th/9603161.

[5] O. Ganor, "A Test Of The Chiral E8 Current Algebra On A 6D Non-Critical String," hep-th/9607020.

[6] A. Klemm, P. Mayr and C. Vafa, "BPS States of Exceptional Non-Critical Strings," hep-th/9607139.

[7] O. Ganor, "Toroidal Compactification of Heterotic 6D Non-Critical Strings Down to Four Dimensions," hep-th/9608109.

[8] N. Seiberg, "Five Dimensional SUSY Field Theories, Non-trivial Fixed Points and String Dynamics," hep-th/9608111.

[9] D. Morrison, N. Seiberg, to appear.

[10] P. Horava and E. Witten, "Heterotic and Type I String Dynamics from ElevenDimensions," Nucl. Phys. B460 (1996)506; "Eleven-dimensional Supergravity on a Manifold with Boundary," hep-th/9603142.

[11] C. Vafa, "Evidence for F-theory," Nucl. Phys. B469 (1996) 403; hep-th/9602022.

[12] J. Polchinski and E. Witten, "Evidence for Heterotic-Type I String Duality," hepth/9510169.

[13] S. Chaudhuri, C. Johnson, and J. Polchinski, "Notes on D-Branes," hep-th/9602052.

[14] M. Bershadsky, V. Sadov and C. Vafa, "D-Branes and Topological Field Theories," Nucl. Phys. B463 (1996) 166.

[15] H. Ooguri and C. Vafa, Nucl. Phys. B463 (1996) 55; hep-th/9511164.

[16] S. Katz and C. Vafa, "Matter From Geometry," hep-th/9606086.

[17] M. Reid, Géométrie Algébrique Angers, (A. Beauville, ed.) (1980) 273.

[18] Griffiths and Harris, Principles of Algebraic Geometry, Wiley-Interscience 1978.

[19] P. S. Aspinwall, Phys. Lett. B371 (1996) 231; hep-th/9511171.

[20] M. Bershadsky, V. Sadov and C. Vafa, Nucl. Phys. B463 (1996) 398; hep-th/9510225.

[21] S. Katz, D. R. Morrison and M. R. Plesser, "Enhanced Gauge Symmetry in Type II String Theory," hep-th/9601108.

[22] A. Klemm and P. Mayr, "Strong Coupling Singularities and Nonabelian Gauge Symmetries in N=2 String Theory," hep-th/9601014. 
[23] P. Berglund, S. Katz, A. Klemm and P. Mayr, "New Higgs Transitions between Dual $\mathrm{N}=2$ String Models," hep-th/9605154.

[24] P.S. Aspinwall and M. Gross, "The $\mathrm{SO}(32)$ Heterotic String on a K3 Surface," hepth/9605131.

[25] M. Bershadsky, K. Intriligator, S. Kachru, D.R. Morrison, V. Sadov and C. Vafa, "Geometric Singularities and Enhanced Gauge Symmetries," hep-th/9605200.

[26] I. Antoniadis, S. Ferrara and T.R. Taylor, "N=2 Heterotic Superstring and its Dual Theory in Five Dimensions," hep-th/9511108.

[27] K. S. Narain, M. H. Sarmadi and E. Witten, Nucl. Phys. B279 (1987)369.

[28] P. Ginsparg, Phys. Rev. D35 (1987)648.

[29] C. Callan, J. Harvey and A. Strominger, Nucl. Phys. B367 (1991)60.

[30] E. Witten, Phys. Lett. B117 (1982) 324.

[31] M. R. Douglas, "Gauge Fields and D-branes," hep-th/9604198.

[32] M. R. Douglas and G. Moore, "D-Branes, Quivers, and ALE Instantons," hepth/9603167.

[33] T. Banks, M. R. Douglas and N. Seiberg, "Probing F-theory with Branes," hepth/9605199.

[34] M. R. Douglas, D. Kabat, P. Pouliot and S. H. Shenker, "D-branes and Short Distances in String Theory," hep-th/9608024.

[35] A. Sen, "F-theory and Orientifolds," hep-th/9605150.

[36] K. Dasgupta and S. Mukhi, "F-Theory at Constant Coupling," hep-th/9606044.

[37] J. A. Minahan and D. Nemeschansky, "An N=2 Superconformal Fixed Point with $E_{6}$ Global Symmetry," hep-th/9608047. 